\title{
Investigating the Effect of a Speckle Pattern on Measurement Uncertainty in a Three-Dimensional Digital Image Correlation (3D-DIC) System
}

\author{
Calvin C. Jee, Gabriel B. P. Salata, David S. Nobes, Kajsa K. Duke \\ Department of Mechanical Engineering \\ University of Alberta \\ Edmonton, Alberta \\ kkduke@ualberta.ca
}

\begin{abstract}
Three-dimensional digital image correlation (3D-DIC) is an imaging technique that uses cameras to measure the surface displacement of a speckled specimen under test loading from which surface strains can be derived. This study aims to investigate the effect of the speckle pattern on the uncertainty in the measurement system. A Monte-Carlo experimental approach is used by uniformly displacing a known speckle pattern by a prescribed amount. This allows the coupled influence of the image collection system, processing and post-processing to be investigated. To minimize the uncertainty of a speckle pattern, it was determined that uniform speckle size of 5-pixel diameter speckles at a density of one speckle per 20 square-pixels is optimal. The methods used to measure and analyze the speckle pattern effects on measurement uncertainty are presented.
\end{abstract}

Keywords-digital image correlation; measurement uncertainty; speckle pattern; strain measurement

\section{INTRODUCTION}

Three-dimensional digital image correlation (3D-DIC) is a measurement technique that uses two cameras in a stereo configuration to measure all three surface displacement components of a specimen [1]. This includes bulk movement of the specimen as well as local movement generated by load and deformation of the sample. Each camera captures two images of a specimen at different stages of the experimental testing. The stereo pairs are used to locate the position of the surface and the difference between successive image pairs is used to calculate the surface displacement.

Some surfaces are intrinsically difficult to track with cameras as they may be smooth, featureless or reflective. This is typically addressed by introducing a random speckle pattern to the surface of the specimen. The approach generates visibly contrasting surface features within the images to be tracked in a manner that is minimally invasive to the specimen surface properties. However, there may be large variations in different speckle patterns due to the difficulty in controlling the application of the speckle. This potentially results in different qualities of data between speckled specimens and even within a single specimen that has varying speckle quality. The objective of this investigation is to determine which characteristics of a speckle pattern most significantly contribute to uncertainty in a 3D-DIC measurement system [2].

\section{METHODOLOGY}

\section{A. Materials}

The image data are captured using a pair of typical commercial machine vision cameras (GX-3300, Allied Vision Prosillica) mounted in a stereo configuration with a $\pm 40^{\circ}$ angle offset from the viewing region-of-interest (ROI) or target, as shown in Figure 1. These are high resolution cameras, each capturing an image $3296 \times 2472$ pixels with an 8-bit resolution. The cameras were equipped with $80 \mathrm{~mm}$ fixed length commercial SLR lenses ( $\# 2.8$, Arax) with a $5^{\circ}$ tilt angle. The tilt allows rotation and overlapping of the focal planes of both cameras to the same ROI. An LED white light source (CN-600SA, Nanguang Photo \& Video Systems Co.) was used for illumination. Using in-house software, cameras were controlled and images were collected and stored for later post processing.

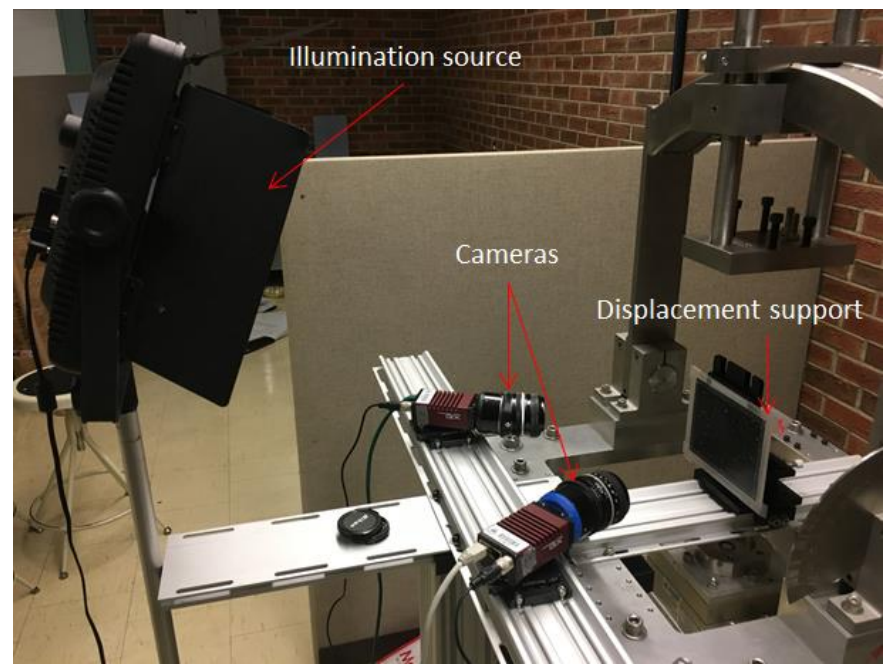

Figure 1. The 3D-DIC measurement system setup for sample testing.

\footnotetext{
Sponsors: NSERC, CFI.
} 
The test specimens were speckle patterns that were generated by a widely used open source software [3] and printed on paper. The software allowed randomized speckle patterns of prescribed speckle densities, speckle sizes and speckle size variations to be generated. Speckle patterns of varying characteristics were printed on printing paper at 1440 dots per inch (dpi). An example comparing the file images of two of the different speckle patterns tested is shown in Figure 2. Note that these images are the same physical size.

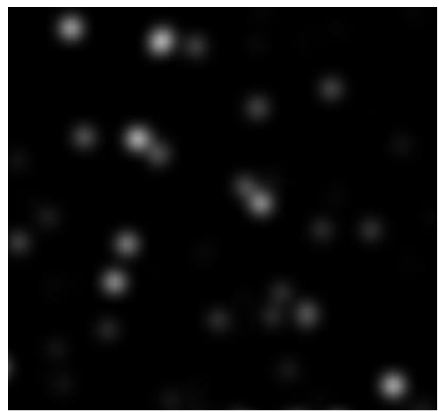

(a)

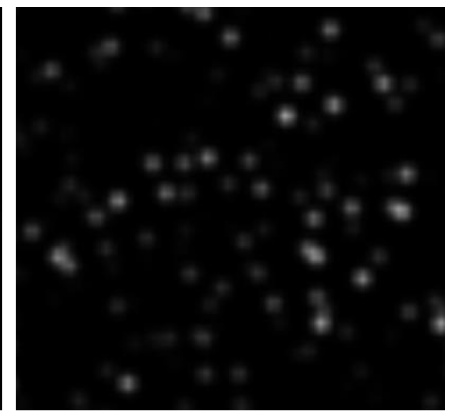

(b)
Figure 2. Comparison of file images of two different speckle patterns: (a) larger speckles at low density, and (b) smaller speckles at high density.

The printed speckle specimens were mounted onto a flat target that was connected to an optomechanical fixture that included a traversing stage. The movements of the stage were controlled and measured by a micrometer allowing movement in only one direction, as shown below in Figure 3. This allowed for the speckle sample to be displaced perpendicularly towards and away from the cameras in a general out-of-plane direction.

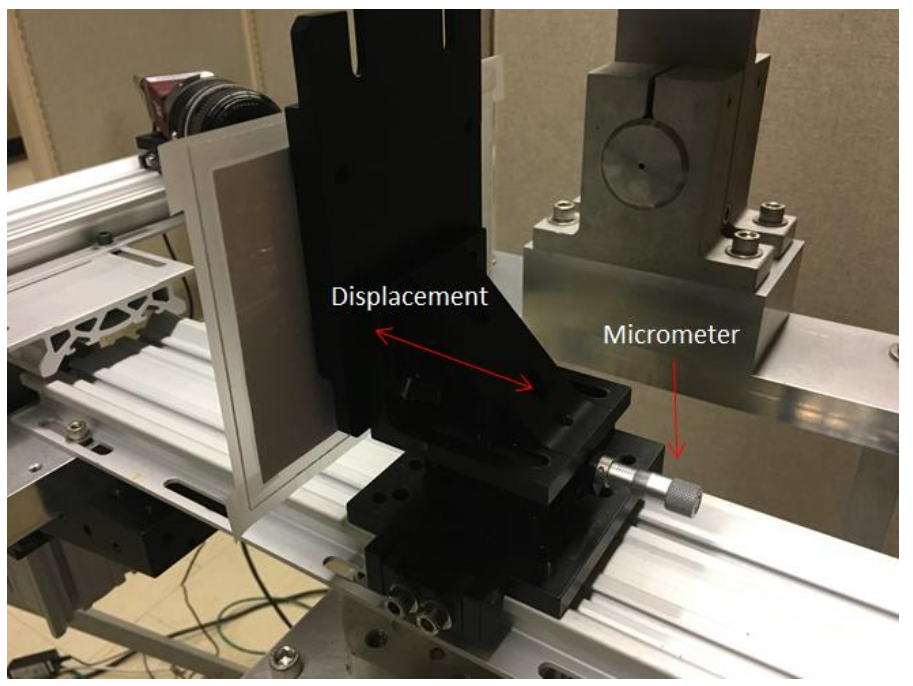

Figure 3. Displacement of micrometer-controlled stage.

\section{B. Digital Image Correlation}

Commercial DIC software (DaVis 8.4, LaVision $\mathrm{GmbH}$ ) was used to analyze the image data. Camera calibration was conducted with a 3D calibration plate (Type 11, LaVision $\mathrm{GmbH}$ ) that contains a known rectangular dot pattern of known dot size and dot spacing at two different surface heights. Each camera captured an image of the calibration plate from their respective fixed positions. The DIC software used these two images to spatially locate the cameras, allowing for the conversion of image data to physical space. The stereo cameras were located in a coordinate system associated with the location and orientation of the calibration target. The calibration target was generally aligned with the coordinate system of the experiment. Images captured post calibration were collected in the coordinate system of cameras, then dewarped and overlapped into the same coordinate system as the calibration target.

The following procedure was used to measure each speckle test specimen. First, a reference image was taken at the position of best focus relative to the cameras. All subsequent displacements were measured in relation to this image. Displacements of $1 \mathrm{~mm}$ steps were made from this point in the out-of-plane direction towards the cameras up to $5 \mathrm{~mm}$. This direction was in the coordinate system of the experiment for which the misalignment with a coordinate system of the cameras was corrected.

After the data were collected, 3D-DIC processing was conducted to extract physical displacements from the image data. To do this, a sub-region, or window, of the image was observed and an image cross-correlation algorithm was conducted between the same window of two different images: a measured displacement image and the reference image. The window of the displaced image was then deformed and compared with the reference image to in an attempt to find a better correlation between the windows of both images. This reoccurred until a better match between the windows could not be found - the final window transformation was the resultant displacement vector of the window. The process was repeated using equally sized and equally spaced windows across the whole image. For all sample displacement images, the result was a displacement field spanning the entire image. The displacement field was then analyzed to determine the uncertainty of the measurement as detailed below.

\section{Design of Experiments}

A $2^{3}$ full factorial experiment based on design of experiments (DOE) was the approach used to quantify how individual characteristics of a speckle pattern affect measurement uncertainty. The responses of this experiment were the two properties used characterize uncertainty: precision and accuracy. The precision was defined as the width of the distribution of measurements in a displacement field, whereas the accuracy was defined as a measure of the difference between the expected displacement, defined by the movement of the micrometer in the experiments, and the average value of all displacement vectors in a displacement field. Thus, the highest quality speckle pattern (i.e., the one with the least uncertainty) was quantifiably the most precise and accurate [4].

The factor combinations for each sample are shown in Table 1. The three input variables were the speckle density, the speckle size and variation in the speckle size. The $2^{3}$ experimental design allowed both main effects and effects of interactions between two factors to be observed. Each input was tested at two levels, and the samples were different combinations of input levels. 
Table 1. Experiment sample factor combinations

\begin{tabular}{|c|c|c|c|}
\hline $\begin{array}{c}\text { Sample } \\
\text { no. }\end{array}$ & $\begin{array}{c}\text { Speckle } \\
\text { density } \\
\left(\# / \text { pixel }^{2} \text { ) }\right.\end{array}$ & $\begin{array}{c}\text { Speckle } \\
\text { diameter } \\
\text { (pixel) }\end{array}$ & $\begin{array}{c}\text { Speckle size } \\
\text { variation } \\
( \pm \text { pixel) }\end{array}$ \\
\hline 1 & 0.20 & 10 & 0 \\
\hline 2 & 0.20 & 10 & 3 \\
\hline 3 & 0.20 & 5 & 0 \\
\hline 4 & 0.20 & 5 & 3 \\
\hline 5 & 0.05 & 10 & 0 \\
\hline 6 & 0.05 & 10 & 3 \\
\hline 7 & 0.05 & 5 & 0 \\
\hline 8 & 0.05 & 5 & 3 \\
\hline
\end{tabular}

As the focus of this study was to investigate the effect of the speckle pattern on the derived displacement, all other parameters were held constant. These included the camera components and positions, the optomechanical components used and the illumination source. The constant parameters of the speckle specimen were the type of paper used and the print settings.

\section{RESULTS AND DISCUSSION}

From the DIC measurements, displacement fields were generated for each measured sample displacements. Because there was no material or local deformation occurring due to loading of the sample, the displacement was expected to be uniform across the entire region and equal to that physically determined by the micrometer. However, this was not the case such as in Figure 4. The measured out-of-plane displacement field, shown in Figure 4(a), shows that the displacement vectors generated by the 3 D-DIC processing for a single speckle pattern were not be identical throughout the entire field. From all measured displacement vectors in the entire field, the probability density function (PDF) in Figure 4 (b) was generated. The difference between the mean and the prescribed displacement was the accuracy of the measurement. The spread of the measurement, quantified by the standard deviation, was the precision. The combination of the two characterize and quantify the uncertainty of the measurement. This measurement procedure was used for every displacement of each speckle sample to compare the uncertainties between speckle patterns of known varying parameters.

After calculating the precision and accuracy of all measurements, the DOE $2^{3}$ full factorial experiment data was analyzed using commercial software (Minitab 18, Minitab Inc.) as summarized in Table 2 below. This statistical analysis used a standard $p$-value $<0.05$ to determine if a main effect or an interaction effect was significant. The analysis showed that for accuracy, all factors had a $p$-value that suggested significance. However, for precision the most significant factors were speckle size variation followed by the effect of the interaction between speckle density and speckle size.

Table 2. Significance of speckle pattern parameters

\begin{tabular}{|c|c|c|}
\hline \multirow{2}{*}{ Factor } & \multicolumn{2}{|c|}{ P-value } \\
\cline { 2 - 3 } & Accuracy & Precision \\
\hline Speckle density & $<\mathbf{0 . 0 0 1}$ & 0.395 \\
\hline Speckle diameter & $\mathbf{0 . 0 0 7}$ & 0.588 \\
\hline Speckle size variation & $<\mathbf{0 . 0 0 1}$ & $\mathbf{0 . 0 2 3}$ \\
\hline $\begin{array}{c}\text { Interaction } \\
\text { (density/diameter) }\end{array}$ & $<\mathbf{0 . 0 0 1}$ & $<\mathbf{0 . 0 0 1}$ \\
\hline $\begin{array}{c}\text { Interaction } \\
\text { (density/size variation) }\end{array}$ & $<\mathbf{0 . 0 0 1}$ & 0.690 \\
\hline $\begin{array}{c}\text { Interaction } \\
\text { (diameter/size variation) }\end{array}$ & $<\mathbf{0 . 0 0 1}$ & 0.454 \\
\hline
\end{tabular}
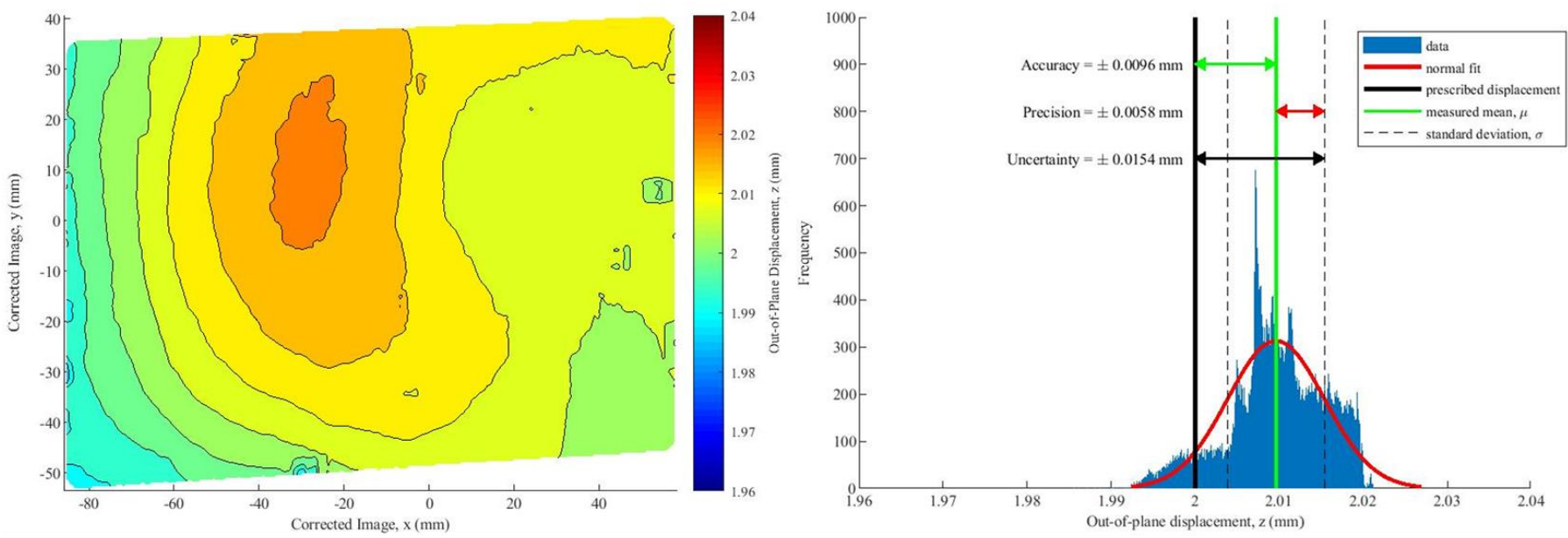

(b)

Figure 4. Displacement of a speckle sample 1 by $2 \mathrm{~mm}$, showing (a) measured displacement vector field and (b) resultant PDF used to determine accuracy and precision of measurement and resultant uncertainty. 
From the means plots of the factors significant to the precision in Figure 5, the most precise speckle pattern had speckle size uniformity and either larger speckles with higher density or smaller speckles with lower density. The difference between either interaction settings was minimal at these levels.

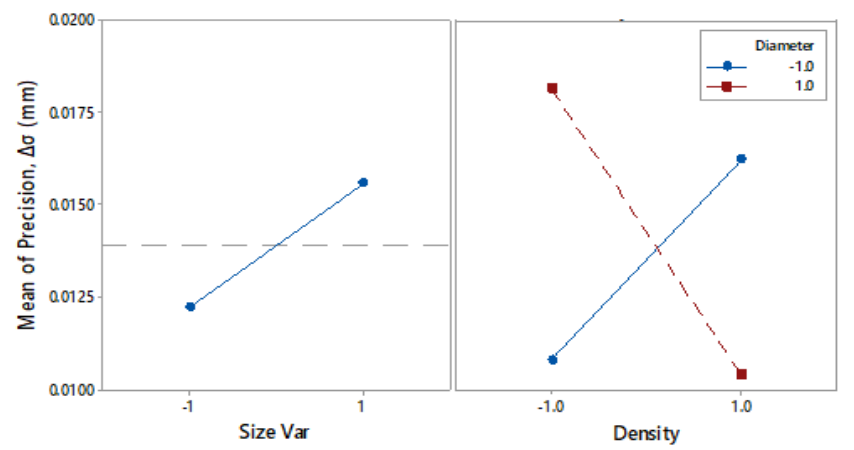

Figure 5. Means plots of effects significant to precision measurement (left speckle size variation; right - speckle density and diameter interaction).

The reason for the reduction of precision from speckle size variation may be due to larger speckles (or larger empty spaces) physically dominating the correlation window. As a result, the smaller speckles have a less significant effect in the DIC process. This means that the displacement measurement is more heavily influenced by fewer particles, resulting in an effectively lower sample size and increasing the standard deviation of the total measurement.

The interaction between speckle density and speckle diameter was the most significant factor regarding precision. Larger speckles at low density may reduce precision similarly to the speckle size variation effect; single large speckles may be too dominant in a single window. Regarding smaller speckles at too high a density, there may be too many speckles in a single correlation window such that they appear as noise that is difficult to track. It also may be a limitation of using speckles printed on paper. The details of a small speckle may be too fine to capture on the prints and in the camera image data. Consequentially, they can appear blurry and may not represent the speckles as accurately as intended. A comparison of a camera image from the experiment and its respective original file is shown in Figure 6.

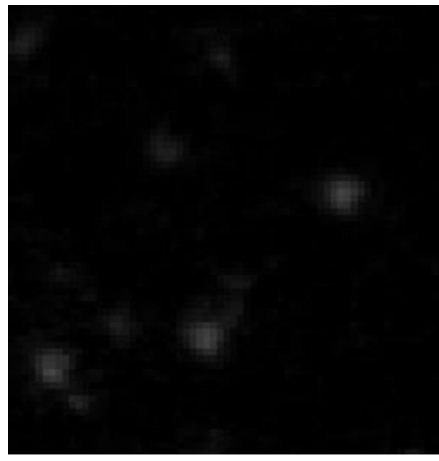

(a)

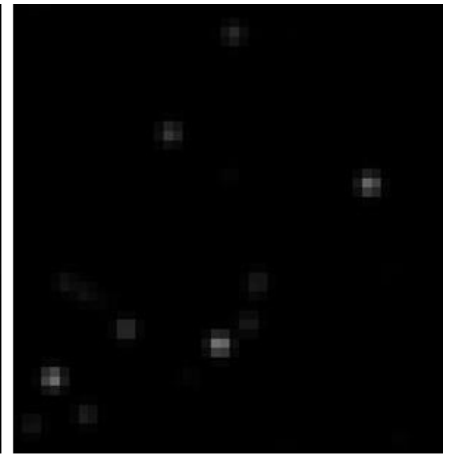

(b)
Figure 6. Comparison of (a) camera image data to (b) original file.
Because all factors were observed as statistically significant regarding accuracy, the pareto chart shown in Figure 7 was used to determine if any factors were more significant than others. The size variation and the interaction between the speckle diameter and density were the most significant in this scenario as well. Because the interactions involving speckle size variation and the other two factors were more significant than the main effects, the remaining factors may be significant by association only. Therefore, heavier emphasis was placed on the main effect of the speckle size variation and the interaction effect of the speckle diameter and density interaction.

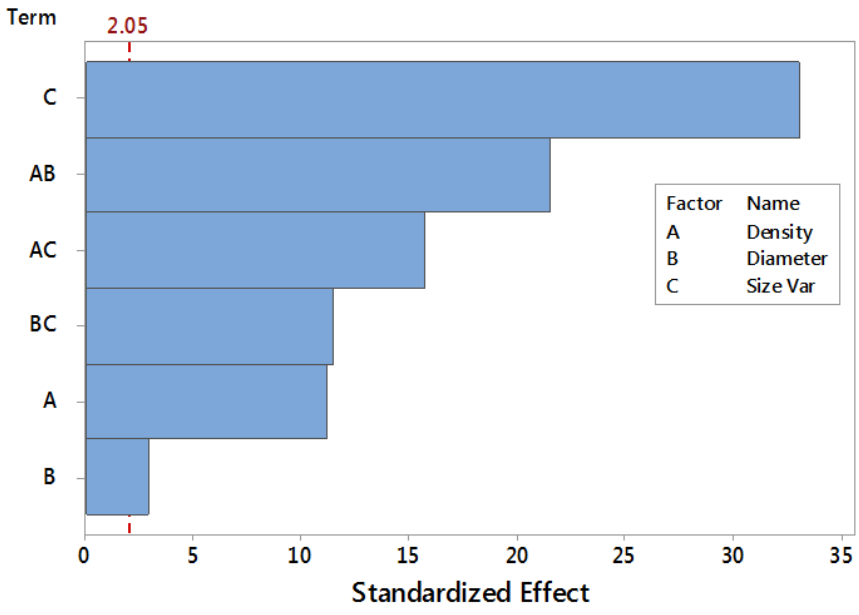

Figure 7. Pareto chart showing factor effects with respect to accuracy.

From Figure 8, the means plots for accuracy showed that the smallest uncertainty resulted from the speckle pattern with lower density, smaller speckle diameters, and speckle size uniformity. Like the statistical analysis of measurement precision, the interaction effect of speckle density and speckle diameter yielded higher accuracy with larger speckles at high density or smaller speckles at low density. However, greater accuracy resulted from the latter. This suggests that the optimal speckle characteristics for minimizing uncertainty (regarding both precision and accuracy) are 5-pixel diameter speckles at a density of 0.05 speckles per square-pixel with no variation in speckle size.

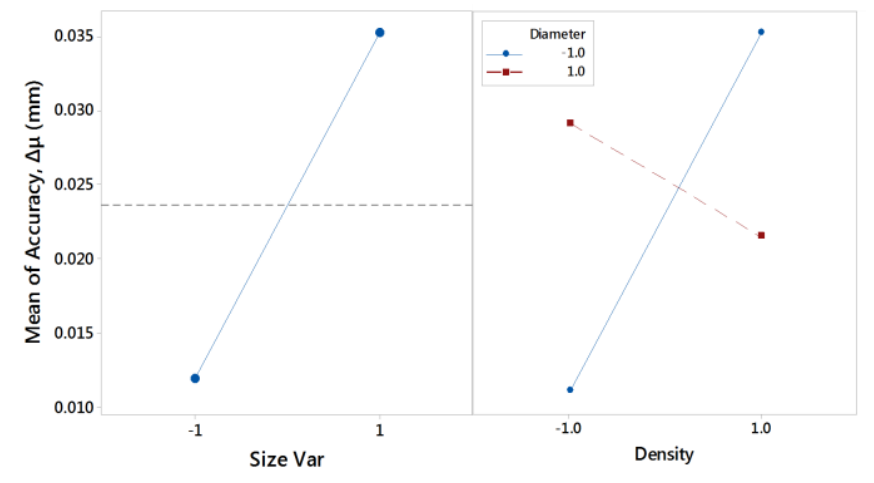

Figure 8. Means plots of effects significant to accuracy measurement (left speckle size variation; right - speckle density and diameter interaction). 
The next step would be to verify the results with physically air-brushed speckled specimens. Along with validating the results, this process would also further investigate what can physically be reproduced and what can practically be applied in an experiment setting using DIC. Additionally, it may be easier to acquire a less blurry image of a speckle pattern with the airbrush method as opposed to the printed paper method, potentially resolving this issue. Through these measures, a greater understanding of the sources of uncertainty and the approaches to limitations within a DIC measurement system can be established.

A major limitation of this study is that the specimen is flat. This serves as a necessary control in the study, but most 3D-DIC applications observe contoured surfaces. 3D surface profiles will be investigated in a future study. Also, this study only measures out-of-plane movement as in-plane movement is expected to be less significant in the overall uncertainty of the measurement of displacement in a stereo setting [5].

\section{CONCLUSION}

This study examined how the characteristics of a speckle pattern affect measurement uncertainty. Using DOE, the effects of input variables and interactions between variables were observed and it was shown that speckle size variation and the effect of the interaction between speckle density and speckle diameter are the most significant effects regarding DIC measurement uncertainty. Within the bounds of this experiment, the optimal speckle pattern was determined to have 5-pixel diameter speckles, a density of one speckle per 20 square-pixel area, and speckle size uniformity. The knowledge from this investigation is significant in understanding the sources of uncertainty within a DIC measurement system, as well as knowing how to effectively approach future applications of DIC.

\section{ACKNOWLEDGMENTS}

The authors acknowledge financial support from Natural Sciences and Engineering Research Council (NSERC) of Canada, the Alberta Ingenuity Fund and the Canadian Foundation for Innovation (CFI).

\section{REFERENCES}

[1] M. N. Helfrick, C. Niezrecki, P. Avitabile, and T. Schmidt, "3D digital image correlation methods for full-field vibration measurement," Mechanical Systems and Signal Processing, 2010.

[2] S. Yaofeng and J. H. L. Pang, "Study of optimal subset size in digital image correlation of speckle pattern images," Optics and Lasers in Engineering, 2007.

[3] W. Thielicke, "PIVlab - particle image velocimetry (PIV) tool," 7 February 2018. [Online]. Available: https://www.mathworks.com/matlabcentral/fileexchange/ 27659-pivlab-particle-image-velocimetry--piv-tool?s_tid=gn_loc_drop. [Accessed 8 February 2018].

[4] H. Haddadi and S. Belhabib, "Use of rigid-body motion for the investigation and estimation of the measurement errors related to digital image correlation technique," Optics and Lasers in Engineering, vol. 46, pp. 185-196, 2008.

[5] N. J. Lawson and J. Wu, "Three-dimensional particle image velocimetry: experimental error analysis of a digital angular stereoscopic system," Measurement Science and Technology, vol. 8, pp. 1455-1464, 1997. 